\title{
Analyzing Water-Supply Situation in the Future
}

\author{
Haoxin Tian ${ }^{1, a}$ \\ ${ }^{1}$ North China Electric Power University, Baoding, Hebei, China \\ a768585058@qq.com
}

Keywords: PCA, Correlation Degree, Time-Series, Gray Markov Model.

\begin{abstract}
In this paper, the water-supply model is established to analyze the ability of a region to provide clean water to meet the needs of its population, the dynamic nature of the factors that affect both supply and demand must be taken into account, the water supply ability in different regions, and predict the water situation in 15 years. The result shows that different regions have different water supply situations, and the water situation in 15 years using our model to predict is not good enough, thus we should to protect water and reusing it to make sure our water supply can be better in the future.
\end{abstract}

\section{Introduction}

Will the world run out of clean water? Nowadays, with the rapid expansion of economic growth, water is increasingly becoming a major constraint that limits the development of human. According to the United Nations, 1.6 billion people (one quarter of the world's population) experience water scarcity, water use has been growing at twice the rate of population over the last century. It means to remind us to protect our water resource and take actions right now. As it's widely approved throughout the world, there are two primary causes for water scarcity: physical scarcity and economic scarcity. In addition, experts think there must be another cause of water scarcity.

Is it possible to provide clean fresh water to all? The supply of water must take the physical availability of water into account. Understanding water availability is an inherently interdisciplinary problem, not only should we attach more importance to population, environment, technology, economy and other important factors, but also we need to consider the related problems tied to the water scarcity.

In order to improve access to clean, fresh water, we propose our solutions.

\section{Symbols and Descriptions}

Table 1. Symbols and Descriptions

\begin{tabular}{cl}
\hline Symbols & \multicolumn{1}{c}{ Descriptions } \\
\hline$\lambda$ & The index of the ability to provide water of \\
$f$ & a region \\
$g$ & The index of Total water supply \\
$p$ & The index of Total water demand \\
\hline
\end{tabular}

\section{Fundamental Assumptions}

The accuracy of our model relies on certain key simplifying assumptions. These assumptions are listed below:

The data we use are all accurate and reliable ;

The factors we use are enough to represent the water supply situation of a region;

We think the variables that can be affected by time and change with no regularities are all dynamic nature;

We don't consider the evaporation of groundwater and the atmospheric water cycle nationwide; 
The water supply ability can be measured by $\left(y_{1}+y_{2}\right) / x$.

\section{Model theory based on the complex network}

The establishment of water-providing model. For building a model that can provide a measure of the ability to provide water of a region,we deal with this problem according to its actual meaning[1] .Thinking of the dynamic nature that affect both supply and demand, we define $\lambda$ to be the index of the ability to provide water , and we apply per capita water supply margin to be $\lambda$,finally we can get a Dynamic Time Series model shown as:

$$
\lambda(t)=\frac{f(t)-g(t)}{P(t)}
$$

Because the factors that affect the $\lambda$ are all random ones, $\lambda$ is also the function of time $t$. And in the formula above, we define:

$$
\begin{aligned}
& f(t)=\sum_{i=1}^{m} \mu_{i} x_{i}(t) \\
& g(t)=\sum_{j=1}^{n} \xi_{j} y_{j}(t)
\end{aligned}
$$

Similarly, we can know from the assumption that $\mu$ and $\xi$ are weights of different factors respectively, so we can know :

$$
\begin{aligned}
& \sum_{i=1}^{m} \mu_{i}=1 \\
& \sum_{j=1}^{n} \xi_{j}=1
\end{aligned}
$$

Select a region for water-analyzing. For solving the problem, we select Shandong province where water is heavily exploited for example. And then use the model built before to get the water supply evaluation index. In addition, we can select another 3 regions for comparison, then by comparison and through the value of main factors respectively, we can explain why and how water is scarce in the region.

After comprehensive analysis based on the data of different regions in some aspects and our model[4], we use PCA method to calculate correlation coefficient matrix and then get contribution rate of each principal component which consists of rainfall, seawater utilization, population, farmland irrigation, industrial waste water and so on. Finally we find the cumulative contribution rate of the top five characteristic roots have reached more than $90 \%$, so we select the five factors for our analysis.

Using our model, we can get the $\lambda$ of different regions in different years, as clearly shown below. The four curves show distinct levels which demonstrate that regions with different water situation shows different water supply ability. 


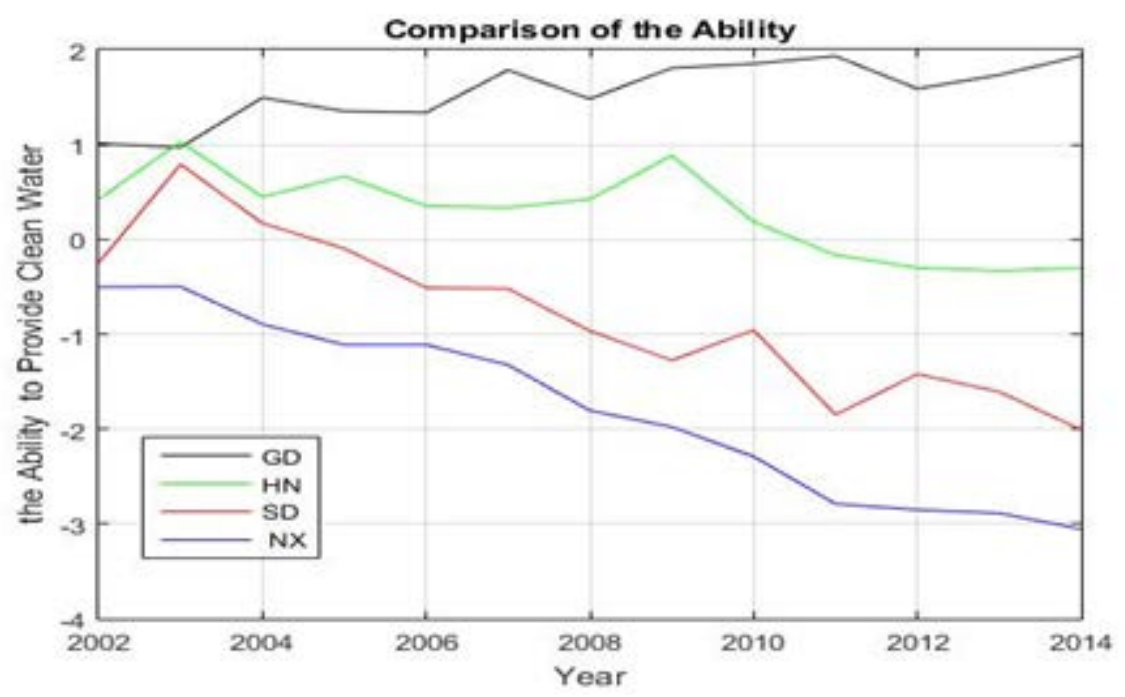

Figure $1 . \lambda$ of 4 regions

(GD stands for Guangdong, HN stands for Hainan,

SD stands for Shandong, and NX stands for Ningxia)

Also, we can find social and environmental reasons :

There are more people with less water in Shandong. And the reason can be generalized as some main aspects below:

First, the rainfall is unbalanced, the flood season lies between June and September, the rainfall of July and August even over $60 \%$ of all rain[2].

Second, the water is unbalanced with geographical feature, the water decrease in turn from southeast to northwest.

Third, the water sources. Yellow River is the main water source to Shandong, so the water decrease of Yellow River has effects on the region; another is the groundwater decrease;

Also, severe water pollution and the flood loss have to account for the situation.

Predicting the water situation in 15 years. Model improvement: We have built our model before, and we use it to measure the water supply ability of different regions successfully, and from Figure 1 we find the value[3] of $\lambda$ and the actual water supply situation fit well.

Now, we use our model to forecast the ability of Shandong and finally we can get the water supply ability situation in 15 years shown as Figure 2:

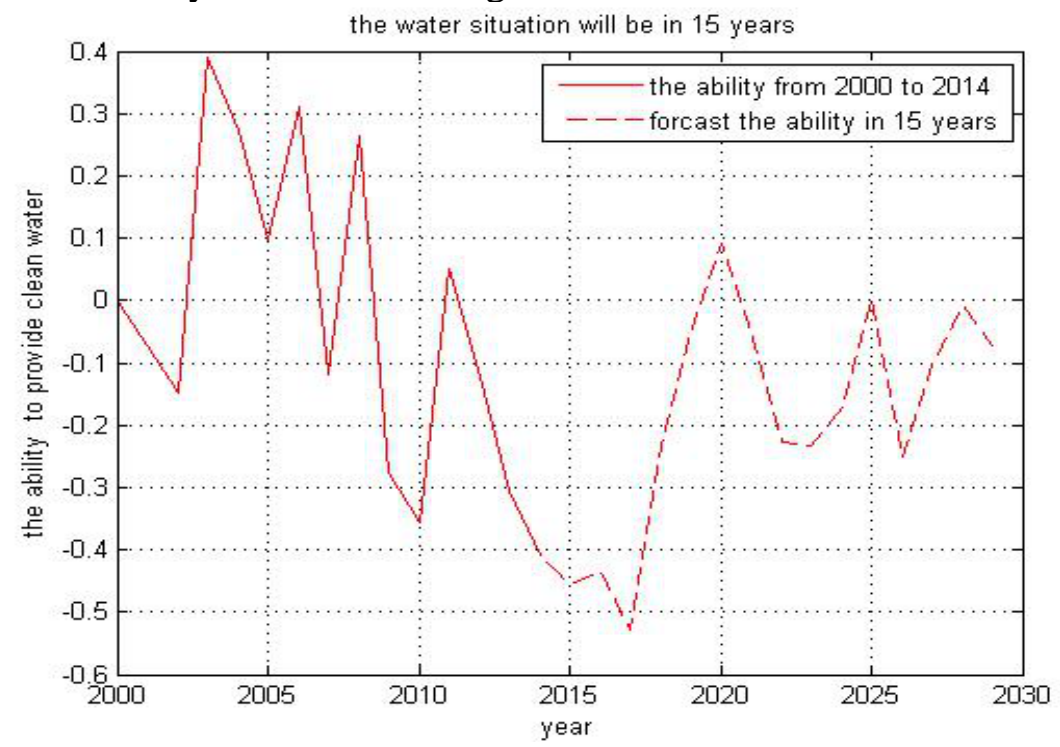

Figure 2. Water ability in 15 years

According to the forecasting curve above, we can clearly find that the value of the ability to provide clean water is less than zero in most years. It shows that water scarcity situation will still exist in 15 years in Shandong province.

Model Testing: Grey Markov Model 
Markov chain can classify and analyze the residual error between the actual value and the predicted value of the gray forecast[5]. Then the predicted values can be modified as following:

First, we need to divide the residual errors sequence into the seven states.

Table 1. Seven states of the residual error divided

\begin{tabular}{c|cll}
\hline State & Range & Meaning & Year \\
\hline E1 & $(-\infty,-0.1)$ & Too large & 2012 \\
E2 & $(-0.1,-0.06)$ & Large & 2010 \\
E3 & $(-0.06,-0.02)$ & Slightly & 2002 \\
& & large & 2009 \\
E4 & $(-0.02,0.02)$ & & 2000 \\
& & Accurate & 2001 \\
E5 & $(0.02,0.06)$ & Slightly & 2004 \\
& & small & 2005 \\
E6 & $(0.06,0.1)$ & Small & 2006 \\
& & & 2008 \\
E7 & $(0.1,+\infty)$ & Too small & 2003 \\
& & & 2011 \\
\hline
\end{tabular}

Then we define $\mathrm{R}$ as the matrix of state transition probability of each residual error.

We have already known that the residual error of 2012 is in the E1 state, and we also can know the residual error of 2013 is in the E2 state based on the state transition probability in the first line of the matrix. Through analysis, beginning from 2013, the residual state of the first k years can be obtained by the state transition probability of the row matrix. We can forecast the water supply ability of a region in year 2013 and 2014 through the optimized Grey - Markov model, and finally we can compare the results with Gray Forecast model to verify the accuracy of this prediction.

Table 2. The comparison between gray forecast model and Markov model

\begin{tabular}{l|llrrc}
\hline Year & $\begin{array}{l}\text { Gray } \\
\text { forecast }\end{array}$ & $\begin{array}{l}\text { Relative } \\
\text { error }\end{array}$ & State & Markov & $\begin{array}{l}\text { Modified } \\
\text { error }\end{array}$ \\
\hline $\mathbf{2 0 1 3}$ & -0.3783 & $16.8 \%$ & E2 & -0.2983 & $3.02 \%$ \\
$\mathbf{2 0 1 4}$ & -0.3395 & $12.4 \%$ & E5 & -0.3295 & $7.01 \%$ \\
\hline
\end{tabular}

The chart above shows that the performance of Gray Markov Model is superior. So we have confidence to use Gray Markov model to predict the water supply capacity index in fifteen years. Meanwhile, the reliability of the first model we made can be tested by comparing the predicted results coming from these two models.

Table 3. The results of three models

\begin{tabular}{l|lccll}
\hline Year & Gray forecast & State & Markov & $\begin{array}{l}\text { Fitted } \\
\text { value }\end{array}$ & $\begin{array}{l}\text { Relative } \\
\text { error }\end{array}$ \\
\hline 2029 & -0.609 & E6 & -0.689 & -0.722 & $4.57 \%$ \\
\hline
\end{tabular}

Finally, we can compare the methods according to their forecasting results, and the results are shown in the Table 4 above. They are almost identical, and the relative error is only $4.57 \%$. So we can use the Gray Markov model to verify the results that we predict through, and it can prove our model made before is efficient to be used to measure the water supply ability of a region.

\section{Conclusions}

The model mentioned above can provide a measure of the ability of a region to provide clean water to meet the needs of its population. Since the model is based on the common model, so it has great practicability and commonality. The model also analyzed the water supply in different regions, and the water supply in 15 years are also measured. PCA and the correlation degree analysis method can reduce the dimension of too many factors, which can reduce the number of variables, so the results we get are objective and reliable. 


\section{References}

[1] An Overview of the State of the World's Fresh and Marine Waters. 2nd Edition, 2008. http://www.unep.org/dewa/vitalwater/index.html;

[2] Liu Hongbin. Countermeasures to solve the problem of water resources shortage in Shandong [J]. land and natural resources research, 1996 (4): Pages 39-42.

[3] Shandong Year book, http://www.stats-sd.gov.cn/.

[4] China Year book , http://www.stats.gov.cn/tjsj/ndsj/.

[5] http://www.yellowriver.gov.cn/other/hhgb/2007.html. 